\title{
Memory, Visual Discrimination Performance, and the Human Hippocampus
}

\author{
Soyun Kim, ${ }^{1}$ Annette Jeneson, ${ }^{2}$ Anna S. van der Horst, ${ }^{1}$ Jennifer C. Frascino, ${ }^{1}$ Ramona 0. Hopkins,,${ }^{3,4}$ and \\ Larry R. Squire $1,2,5,6$ \\ ${ }^{1}$ Department of Psychiatry, ${ }^{2}$ Department of Psychology, ${ }^{3}$ Department of Psychology and Neuroscience Center, Brigham Young University, Provo, Utah \\ 84602, ${ }^{4}$ Department of Medicine, Pulmonary and Critical Care Division, Intermountain Medical Center, Murray, Utah 84143, ${ }^{5}$ Department of \\ Neurosciences, University of California, San Diego, California 92093, and ${ }^{6}$ Veterans Affairs Healthcare System, San Diego, California 92161
}

We evaluated recent proposals that the hippocampus supports certain kinds of visual discrimination performance, for example, when spatial processing is required and the stimuli have a high degree of feature overlap. Patients with circumscribed hippocampal lesions tried to discriminate between images of similar faces or images of similar scenes. In one condition, elements of the stimulus display repeated from trial to trial, and in another condition every trial was unique. In the repeated condition for both faces and scenes, controls gradually improved their performance across testing. In the trial-unique condition, no improvement occurred. The patients were impaired for both faces and scenes in the repeated condition where controls could benefit from learning. However, the patients were fully intact in the trial-unique condition. The results suggest that previous reports of impaired discrimination performance after medial temporal lobe damage may reflect impaired learning rather than impaired visual perception. The findings support the fundamental idea that memory is a distinct cerebral function separable from other perceptual and cognitive abilities.

\section{Introduction}

Considerable evidence indicates that medial temporal lobe structures (MTL) are essential for the formation of declarative memory (Milner, 1972; Gabrieli, 1998; Squire et al., 2004). Studies of both humans and experimental animals over many years have found that bilateral damage to the MTL impairs memory while sparing other perceptual and cognitive functions (Milner et al., 1968; Kensinger et al., 2001; Shrager et al., 2006).

This perspective has been revisited due to interest in the possibility that MTL structures might be important for visual perception in addition to memory (Bussey and Saksida, 2005; Lee et al., 2005a; Baxter, 2009). Specifically, it has been proposed that the perirhinal cortex is needed for visual discrimination performance when the discriminations involve a high degree of feature overlap or feature ambiguity (Bussey et al., 2002; Barense et al., 2005, 2007; Lee et al., 2005b,c). It has also been proposed that the hippocampus is needed when spatial processing is required, for example, in discriminations involving scenes (Lee et al., 2005b,c; Graham et al., 2006).

Recent reviews of this work (Suzuki, 2009, 2010) raised some pertinent issues. First, there is uncertainty about the extent of damage in the studies involving memory-impaired patients. Es-

Received Nov. 10, 2010; revised Dec. 10, 2010; accepted Dec. 28, 2010.

This work was supported by the Medical Research Service of the Department of Veterans Affairs and National Institute of Mental Health Grant 24600. We thank Priya Velu, Kristin Mauldin, Ashley Knutson, Flora Suh, Yael Shrager, and Christine Smith for assistance.

The authors declare no competing financial interests.

Correspondence should be addressed to Larry R. Squire, Veterans Affairs Medical Center 116A, 3550 La Jolla Village Drive, San Diego, CA 92161. E-mail: Isquire@ucsd.edu.

DOI:10.1523/JNEUROSCI.5954-10.2011

Copyright $\odot 2011$ the authors $\quad 0270-6474 / 11 / 312624-06 \$ 15.00 / 0$ timates of brain damage were based on ratings of single sections in each region of interest, and even an analysis of single sections sometimes identified damage outside the structures of interest. Second, impairment might sometimes occur, not because of demands on perception, but because the capacity for working memory has been exceeded such that task performance depends on long-term memory (Shrager et al., 2008; Jeneson et al., 2010).

An additional issue arises because it can be difficult to rule out a role for learning and memory in task performance. Thus, it has been suggested that impairments in experimental animals that have been attributed to a perceptual deficit could have resulted from impaired learning (Hampton, 2005; Squire et al., 2006; Suzuki, 2009). A similar difficulty can occur in studies of memoryimpaired patients with circumscribed hippocampal lesions or hippocampal lesions plus damage to perirhinal cortex. The difficulty arises when the same stimulus set is presented across multiple trials, which allows for the possibility of learning in healthy individuals but not in memory-impaired patients (Barense et al., 2005; Lee et al., 2005a; Graham et al., 2006). The importance of this factor has not been directly evaluated.

Here, we assessed the ability of patients with circumscribed hippocampal lesions to discriminate between different faces or to discriminate between different scenes, all of which had high levels of feature ambiguity. For both faces and scenes, we tested participants in a condition where the same stimulus set was presented on each trial (repeated condition) and also in a condition where the stimulus set was unique on every trial (trial-unique condition). If hippocampal damage impairs the ability to discriminate between feature-ambiguous scenes, then patients should be impaired selectively on the scenes test in both the repeated and 
Table 1. Characteristics of memory-impaired patients

\begin{tabular}{|c|c|c|c|c|c|c|c|c|}
\hline \multirow[b]{2}{*}{ Patient } & \multirow{2}{*}{$\begin{array}{l}\text { Age } \\
\text { (years) }\end{array}$} & \multirow{2}{*}{$\begin{array}{l}\text { Education } \\
\text { (years) }\end{array}$} & \multirow[b]{2}{*}{ WAIS-III IQ } & \multicolumn{5}{|l|}{ WMS-R } \\
\hline & & & & Attention & Verbal & Visual & General & Delay \\
\hline K.E. & 67 & 13.5 & 108 & 114 & 64 & 84 & 72 & 55 \\
\hline L.J. & 71 & 12 & 101 & 105 & 83 & 60 & 69 & $<50$ \\
\hline R.S. & 52 & 12 & 99 & 99 & 85 & 81 & 82 & $<50$ \\
\hline G.W. & 49 & 12 & 108 & 105 & 67 & 86 & 70 & $<50$ \\
\hline J.R.W. & 45 & 12 & 90 & 87 & 65 & 95 & 70 & $<50$ \\
\hline
\end{tabular}

WAIS-III is the Wechsler Adult Intelligence Scale-III and the WMS-R is the Wechsler Memory Scale-Revised. The WMS-R does not provide numerical scores for individuals who score $<50$. IQ scores for J.R.W. and R.S. are from the WAIS-R.

A
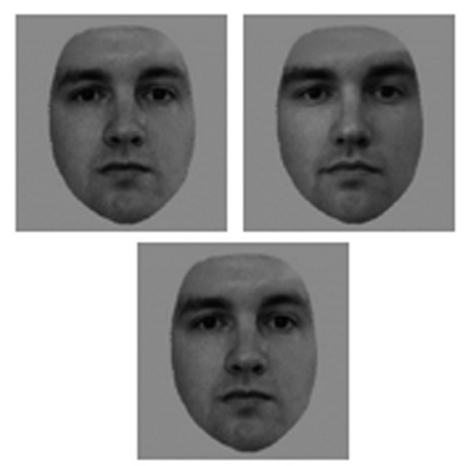

B

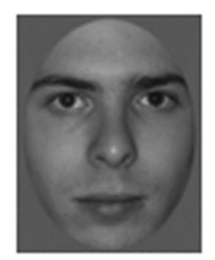

*

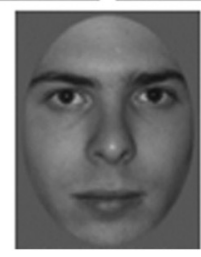

Figure 1. $\boldsymbol{A}, \boldsymbol{B}$, Examples of stimuli used to construct test I $(\boldsymbol{A})$ and test II $(\boldsymbol{B})$. For each trial, two images were displayed side-by-side and participants decided which of these images was more similar to the bottom image. ${ }^{*}$, Correct choice.

trial-unique conditions. Alternatively, if hippocampal damage spares perception but results in a disadvantage whenever the task allows for the possibility of learning, then patients should be impaired in the repeated condition for both faces and scenes, and they should be intact in the trial-unique condition for both faces and scenes.

\section{Materials and Methods}

Participants. Five memory-impaired patients participated (four men) (Table 1), all of whom have bilateral lesions thought to be limited to the hippocampus (CA fields, dentate gyrus, and subicular complex). K.E. became amnesic in 2004 after an episode of ischemia associated with kidney failure and toxic shock syndrome. L.J. (the only female) became amnesic in 1988 during a 6 month period with no known precipitating event. Her memory impairment has been stable since that time. Patients G.W. and R.S. became amnesic in 2001 and 1998, respectively, following drug overdose and associated respiratory failure. J.R.W. became amnesic in 1990 following an anoxic episode associated with cardiac arrest.
Estimates of medial temporal lobe damage were based on quantitative analysis of magnetic resonance images and data from 19 healthy males for the four male patients and 11 healthy females for patient L.J. (Gold and Squire, 2005). K.E., L.J., R.S., G.W., and J.R.W. have an average bilateral reduction in hippocampal volume of $49,46,33,48$, and $44 \%$, respectively (all values $>3$ SDs from the control mean). On the basis of two patients (L.M. and W.H.) with similar bilateral volume loss in the hippocampus for whom detailed postmortem neurohistological information was obtained (Rempel-Clower et al., 1996), this degree of volume loss likely reflects nearly complete loss of hippocampal neurons. The volume of the parahippocampal gyrus in our patients is reduced by 17 , $-8,1,12$, and $6 \%$, respectively (all values within 2 SDs of the control mean).

Additional measurements, based on four controls for each patient, were performed for the frontal lobes, lateral temporal lobes, parietal lobes, occipital lobes, insular cortex, and fusiform gyrus (Bayley et al., 2006). The only volume reduction in these regions $>1.3 \mathrm{SDs}$ of the control mean was the parietal lobe of patient R.S. Nine coronal magnetic resonance images from each patient, together with detailed descriptions of the lesions, can be found in Squire et al. (2010).

Nine healthy volunteers (three female; mean age, 65.7 years; mean education, 15.3 years) also participated.

Materials and procedure. Two tests were constructed for each of two stimulus categories (faces and scenes) (Fig. 1). In each trial, the task was to decide which of two images presented at the top of the screen was more similar to the image presented at the bottom of the screen. One test (test I) consisted of morphed grayscale images of faces and scenes intended to be identical to materials used previously (Graham et al., 2006) (Fig. 1A). The second test (test II) consisted of morphed grayscale images of faces and scenes created with different source materials and different software than were used in test I (Fig. $1 B$ ).

For test I (Fig. 1A), images of faces (Caucasian) and scenes (constructed using Deus Ex SDK editor) were created by gradually morphing one distinct grayscale image into another image across a 100 step series using Morpheus Photo Animator. The two distinct images were labeled 1 and 100 , and the intermediate morphed images were labeled 2-99. Two different morph series for faces and two different series for scenes were created in this way. For each stimulus category (faces and scenes), tests were then constructed using the 30 images from steps $31-45$ and steps 56-70 (Fig. 2). Images 31 and 70 appeared at the top of the screen, and one of the 30 intermediate images appeared at the bottom. These 30 intermediate images allowed for three levels of difficulty: easy, medium, and difficult. The easy condition involved images $31-35$ and $66-70$ that were close to one end of the morphing continuum, the medium condition involved images $36-40$ and $61-65$, and the difficult condition involved images 41-45 and 56-60 that were near the midpoint of the morphing continuum.

For test II, images of faces and scenes (Fig. $1 B$ ) were constructed using a similar method. Morphed images of faces (Caucasian) and scenes (constructed using Punch! Home Design Architectural Series) were created by gradually morphing one image into another image across a 100-step series using Abrosoft FantaMorph 4.0. Next, images 31 and 70 in the 100 -image series were used to create a second continuum of 30 morphs. For each stimulus category, tests were then constructed using these 30 images. Images 1 and 30 appeared at the top of the screen, and one of the 30 images in the continuum appeared below. The lower images allowed 


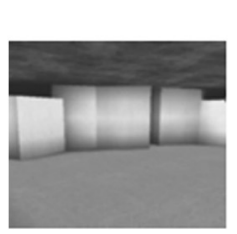

31

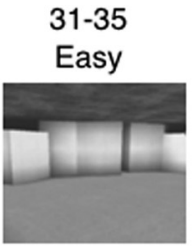

33

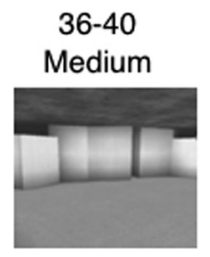

38

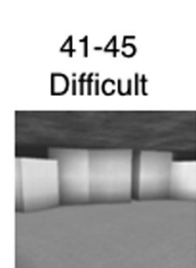

43

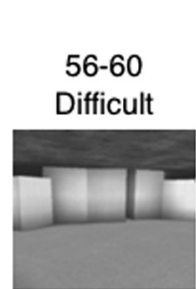

58

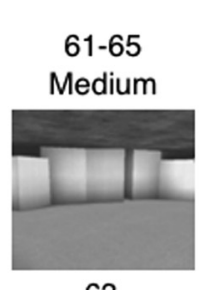

63
66-70

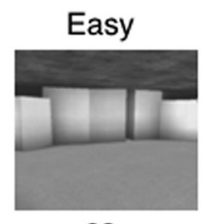

68

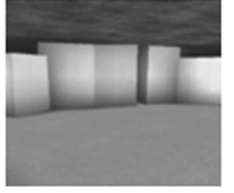

70

Figure 2. Morphed images were created by gradually morphing one distinct image into another distinct image across a 100-step series. Test I, illustrated here, was constructed using the 30 images from steps $31-45$ and $56-70$. The numbers below each image indicate its position in the morph series. The materials for faces and for test II were created using a similar method. For testing with the material illustrated here, images 31 and 70 appeared at the top of the screen and one of the intermediate images appeared at the bottom. How close the bottom image was to image 31 or 70 determined whether the judgment was easy, medium, or difficult.

for three levels of difficulty [images $1-5$ and 26-30 (easy), images 6-10 and 21-25 (medium), and images 11-20 (difficult)].

Both tests (I and II) were presented in a repeated condition as well as in a trial-unique condition (Fig. 3). Each trial consisted of a display of three images from a morph series presented on a monotone gray background. The repeated condition was designed to match the procedure used previously with these materials (Graham et al., 2006). Accordingly, in this condition, tests I and II each consisted of two blocks of 90 trials with faces and two blocks of 90 trials with scenes for a total of 360 trials for faces and 360 trials for scenes $(2$ tests $\times 2$ blocks $\times 90$ trials). For each 90 -trial block, 30 trials were given at each level of difficulty (easy, medium, and difficult). Note that for all the trials in each 90-trial block, the two images at the top of the screen were always the same and were taken from the same morphing series. In addition, the same lower image was repeated three times in each 90-trial block.

In the trial-unique condition, the two images at the top of the screen were unique in every trial and were taken from steps 31 and 70 of a unique morphing series. Each test (I and II) consisted of one block of 60 trials with faces and one block of 60 trials with scenes for a total of 120 trials for faces and 120 trials for scenes (2 tests $\times 1$ block $\times 60$ trials). For each block of 60 trials, 20 trials were given at each level of difficulty.

In test I, each face subtended a visual angle of $4^{\circ}$ in width, and each scene subtended a visual angle of $6^{\circ}$ in width. The two images displayed side-by-side were $10^{\circ}$ apart (center to center) and the lower image was $7.5^{\circ}$ below the two top images. In test II, each face subtended a visual angle of $4^{\circ}$ in width, and each scene subtended a visual angle of $10^{\circ}$ in width. The two images displayed side-by-side were $15^{\circ}$ apart (center to center), and the bottom image was presented $13^{\circ}$ below the two top images.

Faces and scenes were presented separately. Testing occurred in four separate sessions ( 2 tests $\times 2$ conditions) scheduled at least $7 \mathrm{~d}$ apart in the following order: (1) test I, repeated condition; (2) test II, trial-unique condition; (3) test II, repeated condition; and (4) test I, trial-unique condition. For each test, approximately half of the participants were tested first with faces, and the others were tested first with scenes. In each of the four test sessions, trials were presented in a different, pseudorandom order for each participant, with the constraint that no more than three trials in a row could be of the same level of difficulty. Trials were self-paced, though participants were encouraged to make decisions as

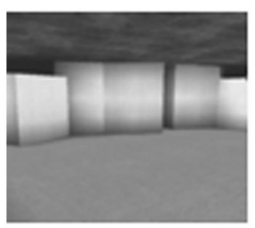

\section{Repeated}
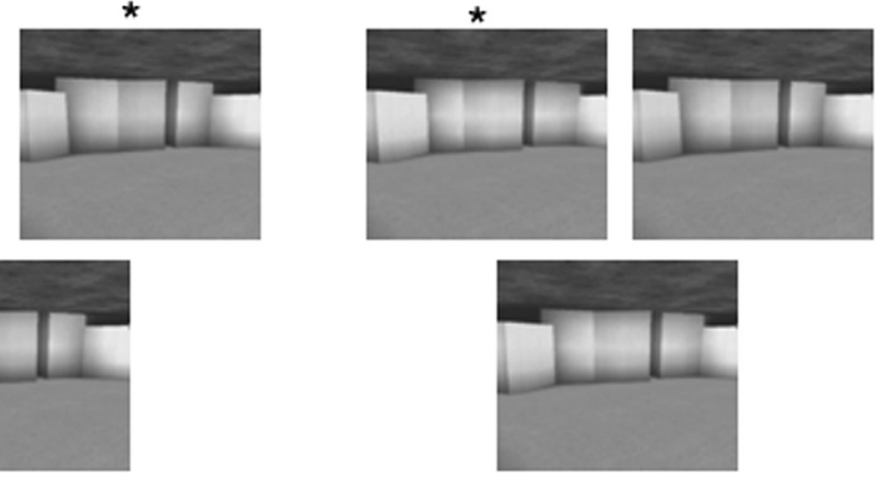

\section{Trial Unique}
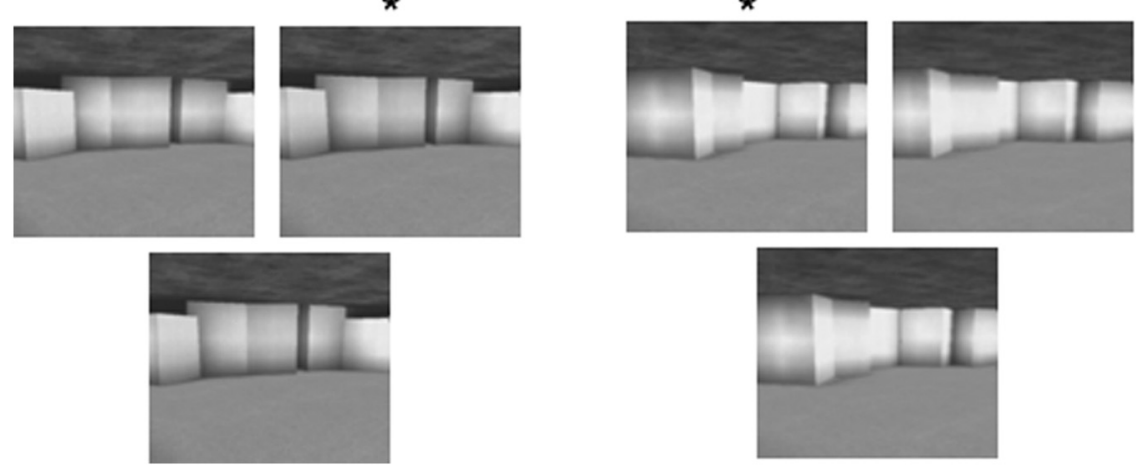

Figure 3. Examples of two trials from the repeated condition of test I and two trials from the trial-unique condition of test I. In both conditions, two images appeared at the top of the display and an intermediate image appeared at the bottom. Note that in the two trials illustrated for the repeated condition, the two top images are identical. In this condition, the same pair of top images was used across 90 trials and an intermediate image from the same morph series appeared at the bottom (i.e., all the displays in a 90 -trial block consisted of images from the same morph series). In the two trials illustrated for the trial-unique condition, the two top images are different. In this condition, the images presented in every trial came from a different morph series and each display was presented only once. Thus, each display was unique for every trial. * , Correct choice.

quickly as possible without compromising accuracy. Feedback was not provided. Before each test, participants completed 16 practice trials to introduce the procedure. For these practice trials, a yellow square and a red square were presented side-by-side and a morph (or blend) of these two colors was presented at the bottom.

\section{Results}

The overall scores for tests I and II were approximately the same ( $3.1 \%$ difference), and the scores for the two tests were therefore combined. Figure 4 shows the scores of memory-impaired pa- 

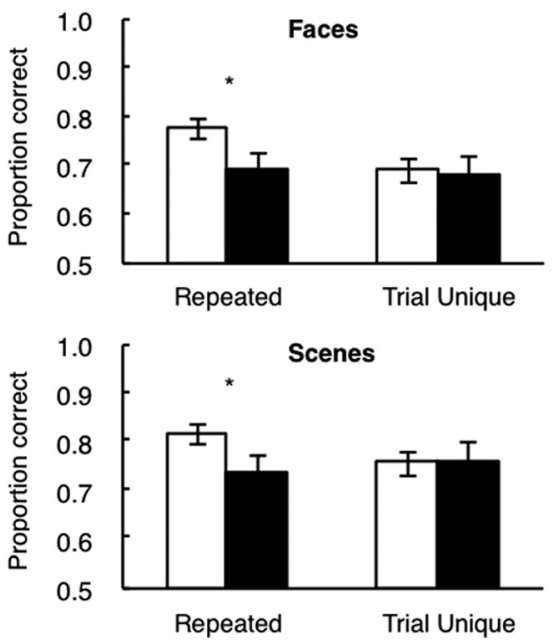

Figure 4. Performance of controls (white bars) and patients with hippocampal lesions (black bars) on a perceptual judgment task for faces (top) and scenes (bottom). The patients were impaired when displays were repeated from trial to trial but intact when every display was unique. ${ }^{*} p<0.05$ compared with the control group. Error bars show SEM.

Separate analyses of the data for tests I and II revealed similar effects, though the group $\times$ condition interaction reached significance only for scenes in test I $(p<0.03)$. Nonetheless, the results for both tests and each stimulus condition (faces and scenes) showed similar trends of impaired performance by the patients in the repeated condition and intact performance by the patients in the trial-unique condition.

The finding that controls were advantaged in the repeated condition suggests that they were able to benefit from the fact that the two images at the top of the display were identical across all 90 trials. If so, the controls, but not the patients, should have exhibited gradually improved performances, i.e., learning, across the 90 trials of testing. Figure 5, $A$ and $C$, shows the scores for each block of 10 trials in the repeated condition. As expected, the controls exhibited learning when tested with faces (positive linear trend, $F_{(1,8)}=5.7, p<0.05$ ) and when tested with scenes (positive linear trend, $\left.F_{(1,8)}=16.2, p<0.01\right)$. These learning effects were also evident within the first 60 trials $(p s<0.05$ ). In contrast, the patients did not learn in the repeated condition, performing similarly across trial blocks for both faces and scenes (all $p s>0.2$ ). In the trial-unique condition, the entire display was unique for each of 60 trials and, therefore, there was no basis for trial-to-trial learning about the displays. Figure $5, B$ and $D$, confirm that controls and patients exhibited no evidence of learning on either the faces test or the scenes test (all ps $>0.5$ ).

We next examined the effect of difficulty level on performance. Overall, performance on the faces test was $84.8,77.8$, and $69.3 \%$ correct across the three levels of difficulty (easy, medium, and hard, respectively). For scenes, the corresponding scores were 79.5, 74.3, and $60.7 \%$ correct, respectively. Separate ANOVAs for faces and scenes documented the marked effect of difficulty level on performance ( $p s<0.001)$. Importantly, the difference between control and patient scores was similar across difficulty levels and there were no group $x$ difficulty interactions $(p s>0.4)$. In the repeated condition, separate comparisons at each difficulty level revealed an impairment for the patients at the medium level of difficulty for faces and at the easy and medium levels for scenes $(t s>2.1, p s<$ $0.05)$. In contrast, in the trial-unique con-

tients and controls on the faces test and on the scenes test in each of the two conditions (repeated and trial-unique). For faces, an ANOVA revealed an effect of condition $\left(F_{(1,12)}=8.9, p<0.05\right)$, no effect of group $\left(F_{(1,12)}=1.6, p>0.20\right)$, and a group $\times$ condition interaction $\left(F_{(1,12)}=5.6, p<0.05\right)$. The interaction reflects the fact that controls performed better than patients in the repeated condition ( 77.5 vs $68.6 \%$ correct, $\left.t_{(12)}=2.2, p<0.05\right)$, whereas the two groups performed almost identically in the trialunique condition ( 68.9 vs $67.7 \%$ ).

For scenes, there was no effect of condition $\left(F_{(1,12)}=1.1, p>\right.$ $0.3)$ or group $\left(F_{(1,12)}=1.5, p>0.2\right)$, but there was a group $\times$ condition interaction $\left(F_{(1,12)}=5.7, p<0.05\right)$. The interaction reflects the fact that controls performed better than patients in the repeated condition ( 81.6 vs $73.4 \%$ correct, $t_{(12)}=2.8, p<$ 0.05 ), whereas the two groups performed almost identically in the trial-unique condition ( 75.6 vs $75.8 \%$ correct). dition, none of the comparisons at any difficulty level approached significance for either faces or scenes (all ps > 0.15).

Last, we examined the response times of patients and controls for each test (faces and scenes) and for each condition (repeated and trial-unique). There were two principal findings. First, the patients and controls had very similar response times. Second, for both groups and for both tests, response times were considerably faster in the repeated condition than in the trial-unique condition. Thus, for faces, an ANOVA yielded an effect of condition $\left(F_{(1,12)}=45.1, p<0.001\right)$ but no effect of group $(F=0.6)$ and no group $\times$ condition interaction $(F=0.0)$. The mean response time for controls was $3.0 \pm 0.3 \mathrm{~s}$ in the repeated condition and $4.9 \pm 0.4 \mathrm{~s}$ in the trial-unique condition. For patients, the corresponding values were $2.5 \pm 0.6 \mathrm{~s}$ in the repeated condition and $4.4 \pm 0.5 \mathrm{~s}$ in the trial-unique condition. For scenes, an ANOVA also yielded an effect of condition $\left(F_{(1,12)}=82.9, p<0.001\right)$ but 
no effect of group $(F=0.0)$ and no group $\times$ condition interaction $(F=0.6)$. The mean response time for controls was $2.2 \pm 0.2 \mathrm{~s}$ in the repeated condition and $5.7 \pm 0.6 \mathrm{~s}$ in the trial-unique condition. For patients, the corresponding values were $2.4 \pm 0.5 \mathrm{~s}$ and $5.5 \pm 0.6 \mathrm{~s}$.

Interestingly, in the repeated condition, the response time of both controls and patients improved during testing (Fig. 6A,C). For faces, the effect was significant for both groups $\left(F_{(1,8)}>11.8\right.$, $p s<0.03)$. For scenes, the effect was significant in controls $\left(F_{(1,8)}=89.3, p<\right.$ $0.01)$ and marginally significant for patients $\left(F_{(1,4)}=5.5, p<0.08\right)$. In contrast, for the trial-unique condition, response time was similar across all 60 trials (all ps $>0.2$ ).

\section{Discussion}

Memory-impaired patients with circumscribed hippocampal damage were given difficult visual discrimination tests for faces and scenes in both a repeated and a trial-unique condition. In the repeated condition, the top two images in the display were the same in every trial. For the trial-unique condition, a different display was presented on every trial (Fig. 3). In the repeated condition, the patients were impaired at discriminating both faces and scenes (Fig. 4). In contrast, in the trial-unique condition, patients and controls performed similarly for both faces and scenes. The fact that controls performed better than patients only in the repeated condition suggested that controls (but not patients) were able to learn about the displays across trials and in that way gain an advantage over the patients. Consistent with this idea, we found that controls improved their performance gradually across the 90 trials of testing for both faces and scenes, whereas patients did not improve (Fig. $5 \mathrm{~A}, \mathrm{C}$ ). Furthermore, improvement across testing for the controls was evident only in the repeated condition, not in the trial-unique condition (Fig. 5B,D).

Response times also improved across testing in the repeated condition but not in the trial-unique condition (Fig. 6). However, unlike discrimination accuracy itself, which improved for controls but not for patients, response times improved for both groups (Fig. 6A,C). This finding is reminiscent of an early observation of the noted patient H.M. (Corkin, 1965). While attempting to learn a path through a tactual maze, H.M. exhibited no improvement in choice accuracy but the time needed to complete each trial markedly decreased across testing.

Our findings suggest that memory-impaired patients are disadvantaged on visual discrimination tasks whenever there is the possibility of learning about the test displays across trials. Previous studies of patients with hippocampal damage, or larger lesions that include perirhinal cortex, often involved visual discrimination tasks in which the same stimulus set was repeated across multiple trials (Barense et al., 2005; Lee et al., 2005c; Graham et al., 2006). In one study (Barense et al., 2005), object discrimination learning was tested with stimulus pairs that had different degrees of feature ambiguity. Whereas patients with hippocampal lesions performed normally at all levels of feature ambiguity, patients with damage to perirhinal cortex were impaired when the objects had high feature ambiguity. In
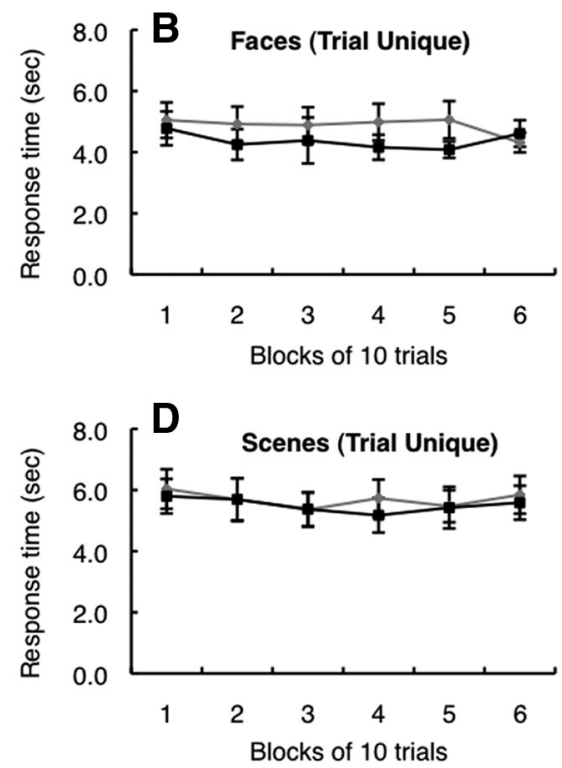
tainty about the locus of damage in some of the patients who have been studied. For example, two of the patients with hippocampal lesions studied previously (Lee et al., 2005b; Barense et al., 2007) also had significant additional damage to the parahippocampal gyrus (Lee et al., 2005c; Graham et al., 2010).

In summary, we assessed visual discrimination performance in patients with hippocampal lesions. Most previous studies used stimulus displays that were repeated across trials and that allowed for the possibility that, in the absence of memory impairment, performance might improve across testing as the result of gradual learning. We tested discrimination ability for faces and scenes when the stimulus displays were repeated from trial to trial and also when each trial was unique. In the repeated condition for both faces and scenes, controls gradually improved their performance across testing. In the trial-unique condition, no improvement occurred. Patients with hippocampal lesions were impaired in the repeated condition, where controls could benefit from learning, but patients were fully intact in the trial-unique condition. The results do not support the idea that visual perception is impaired after hippocampal lesions and suggest that poor performance in many earlier studies resulted from impaired learning rather than impaired perception.

\section{References}

Barense MD, Bussey TJ, Lee AC, Rogers TT, Davies RR, Saksida LM, Murray EA, Graham KS (2005) Functional specialization in the human medial temporal lobe. J Neurosci 25:10239-10246.

Barense MD, Gaffan D, Graham KS (2007) The human medial temporal lobe processes online representations of complex objects. Neuropsychologia 45:2963-2974.

Baxter MG (2009) Involvement of medial temporal lobe structures in memory and perception. Neuron 61:667-677.

Bayley PJ, Hopkins RO, Squire LR (2006) The fate of old memories after medial temporal lobe damage. J Neurosci 26:13311-13317.

Bussey TJ, Saksida LM (2005) Object memory and perception in the medial temporal lobe: an alternative approach. Curr Opin Neurobiol 15: $730-737$.

Bussey TJ, Saksida LM, Murray EA (2002) Perirhinal cortex resolves feature ambiguity in complex visual discriminations. Eur J Neurosci 15:365-374.

Corkin S (1965) Tactually-guided maze learning in man: effects of unilateral cortical excisions and bilateral hippocampal lesions. Neuropsychologia 3:339-351.

Cowan N (2001) The magical number 4 in short-term memory: a reconsideration of mental storage capacity. Behav Brain Sci 24:87-114.

Fukuda K, Awh E, Vogel EK (2010) Discrete capacity limits in visual working memory. Curr Opin Neurobiol 20:177-182.

Gabrieli JD (1998) Cognitive neuroscience of human memory. Annu Rev Psychol 49:87-115.
Gold JJ, Squire LR (2005) Quantifying medial temporal lobe damage in memory-impaired patients. Hippocampus 15:79-85.

Graham KS, Scahill VL, Hornberger M, Barense MD, Lee AC, Bussey TJ, Saksida LM (2006) Abnormal categorization and perceptual learning in patients with hippocampal damage. J Neurosci 26:7547-7554.

Graham KS, Barense MD, Lee AC (2010) Going beyond LTM in the MTL: a synthesis of neuropsychological and neuroimaging findings on the role of the medial temporal lobe in memory and perception. Neuropsychologia 48:831-853.

Hampton RR (2005) Monkey perirhinal cortex is critical for visual memory, but not for visual perception: reexamination of the behavioural evidence from monkeys. Q J Exp Psychol B 58:283-299.

Jeneson A, Mauldin KN, Squire LR (2010) Intact working memory for relational information after medial temporal lobe damage. J Neurosci 30: 13624-13629.

Kensinger EA, Ullman MT, Corkin S (2001) Bilateral medial temporal lobe damage does not affect lexical or grammatical processing: evidence from amnesic patient H.M. Hippocampus 11:347-360.

Lee AC, Barense MD, Graham KS (2005a) The contribution of the human medial temporal lobe to perception: bridging the gap between animal and human studies. Q J Exp Psychol B 58:300-325.

Lee AC, Buckley MJ, Pegman SJ, Spiers H, Scahill VL, Gaffan D, Bussey TJ, Davies RR, Kapur N, Hodges JR, Graham KS (2005b) Specialization in the medial temporal lobe for processing of objects and scenes. Hippocampus 15:782-797.

Lee AC, Bussey TJ, Murray EA, Saksida LM, Epstein RA, Kapur N, Hodges JR, Graham KS (2005c) Perceptual deficits in amnesia: challenging the medial temporal lobe 'mnemonic' view. Neuropsychologia 43:1-11.

Milner B (1972) Disorders of learning and memory after temporal lobe lesions in man. Clin Neurosurg 19:421-446.

Milner B, Corkin S, Teuber HL (1968) Further analysis of hippocampal amnesic syndrome: 14-year follow-up study of H.M. Neuropsychologia 6:215-234.

Rempel-Clower NL, Zola SM, Squire LR, Amaral DG (1996) Three cases of enduring memory impairment after bilateral damage limited to the hippocampal formation. J Neurosci 16:5233-5255.

Shrager Y, Gold JJ, Hopkins RO, Squire LR (2006) Intact visual perception in memory-impaired patients with medial temporal lobe lesions. J Neurosci 26:2235-2240.

Shrager Y, Levy DA, Hopkins RO, Squire LR (2008) Working memory and the organization of brain systems. J Neurosci 28:4818-4822.

Squire LR, Stark CE, Clark RE (2004) The medial temporal lobe. Annu Rev Neurosci 27:279-306.

Squire LR, Shrager Y, Levy DA (2006) Lack of evidence for a role of medial temporal lobe structures in visual perception. Learn Mem 13:106-107.

Squire LR, van der Horst AS, McDuff SG, Frascino JC, Hopkins RO, Mauldin KN (2010) Role of the hippocampus in remembering the past and imagining the future. Proc Natl Acad Sci U S A 107:19044-19048.

Suzuki WA (2009) Perception and the medial temporal lobe: evaluating the current evidence. Neuron 61:657-666.

Suzuki WA (2010) Untangling memory from perception in the medial temporal lobe. Trends Cogn Sci 14:195-200. 